\title{
Erratum: Systematic decomposition of the neutrinoless double beta decay operator
}

\author{
Florian Bonnet, ${ }^{a}$ Martin Hirsch, ${ }^{b}$ Toshihiko Ota ${ }^{c, d}$ and Walter Winter ${ }^{a}$ \\ ${ }^{a}$ Institut für Theoretische Physik und Astrophysik, Universität Würzburg, \\ 97074 Würzburg, Germany \\ ${ }^{b}$ AHEP Group, Instituto de Física Corpuscular - C.S.I.C./Universitat de València, \\ Edificio de Institutos de Paterna, Apartado 22085, 46071 València, Spain \\ ${ }^{c}$ Max-Planck-Institut für Physik (Werner-Heisenberg-Institut), \\ Föhringer Ring 6, 80805 München, Germany \\ ${ }^{d}$ Department of Physics, Saitama University, \\ Shimo-Okubo 255, 338-8570 Saitama-Sakura, Japan \\ E-mail: florian.bonnet@physik.uni-wuerzburg.de, mahirsch@ific.uv.es, \\ toshi@mppmu.mpg.de, winter@physik.uni-wuerzburg.de
}

ERRATUM TO: JHEP03(2013)055

Keywords: Beyond Standard Model, Neutrino Physics

ArXiv EPRINT: 1212.3045

We have discovered a few misprints in tables 8 and 9 of the paper. Some quantum numbers for the intermediate scalar or fermion where incorrectly given. The correct quantum numbers are given in table 1 , where the corrections are emphasized in red color.

In addition, in table 9 two possible decompositions were not listed by accident. The missing decompositions correspond to two cases in decomposition 4-i, BL\#11, and are given in table 2 .

Open Access. This article is distributed under the terms of the Creative Commons Attribution License (CC-BY 4.0), which permits any use, distribution and reproduction in any medium, provided the original author(s) and source are credited. 


\begin{tabular}{|c|c|c|c|c|c|}
\hline Decomp: & Operator & BL & $S$ & $\Psi$ & $S^{\prime}$ \\
\hline 2-iii-b & $\left(d_{R} \overline{e_{L}}\right)\left(d_{L}\right)\left(\overline{u_{R}}\right)\left(\overline{u_{R} e_{R}}\right)$ & $\# 20$ & $\begin{array}{l}(\overline{\mathbf{3}}, \mathbf{2})_{-1 / 6} \\
(\overline{\mathbf{3}}, \mathbf{2})_{-1 / 6}\end{array}$ & $\begin{array}{l}(\mathbf{3}, \mathbf{1})_{-1 / 3} \\
(\overline{\mathbf{6}}, \mathbf{1})_{-1 / 3}\end{array}$ & $\begin{array}{l}(\overline{\mathbf{3}}, \mathbf{1})_{+1 / 3} \\
(\overline{\mathbf{3}}, \mathbf{1})_{+1 / 3}\end{array}$ \\
\hline $5-\mathrm{i}$ & $\begin{array}{l}\left(\overline{u_{L} e_{L}}\right)\left(d_{R}\right)\left(d_{R}\right)\left(\overline{u_{L} e_{L}}\right) \\
\left(\overline{u_{R} e_{R}}\right)\left(d_{R}\right)\left(d_{R}\right)\left(\overline{u_{L} e_{L}}\right) \\
\left(\overline{u_{R} e_{R}}\right)\left(d_{R}\right)\left(d_{R}\right)\left(\overline{u_{R} e_{R}}\right)\end{array}$ & $\begin{array}{l}\# 11 \\
\# 19\end{array}$ & $\begin{array}{l}(\mathbf{3}, \mathbf{3})_{-1 / 3} \\
(\mathbf{3}, \mathbf{3})_{-1 / 3} \\
(\mathbf{3}, \mathbf{1})_{-1 / 3} \\
(\mathbf{3}, \mathbf{1})_{-1 / 3} \\
(\mathbf{3}, \mathbf{1})_{-1 / 3} \\
(\mathbf{3}, \mathbf{1})_{-1 / 3}\end{array}$ & $\begin{array}{l}(\mathbf{1}, \mathbf{3})_{0} \\
(\mathbf{8}, \mathbf{3})_{0} \\
(\mathbf{1}, \mathbf{1})_{0} \\
(\mathbf{8}, \mathbf{1})_{0} \\
(\mathbf{1}, \mathbf{1})_{0} \\
(\mathbf{8}, \mathbf{1})_{0}\end{array}$ & $\begin{array}{l}(\overline{\mathbf{3}}, \mathbf{3})_{+1 / 3} \\
(\overline{\mathbf{3}}, \mathbf{3})_{+1 / 3} \\
(\overline{\mathbf{3}}, \mathbf{1})_{+1 / 3} \\
(\overline{\mathbf{3}}, \mathbf{1})_{+1 / 3} \\
(\overline{\mathbf{3}}, \mathbf{1})_{+1 / 3} \\
(\overline{\mathbf{3}}, \mathbf{1})_{+1 / 3}\end{array}$ \\
\hline
\end{tabular}

Table 1. Misprints in tables 8 and 9. The corrected quantum numbers are emphasized in red colour.

\begin{tabular}{|ccccccc|}
\hline$\#$ & Operators & BL & $S$ & $\psi$ & $S^{\prime}$ & Basis op. \\
\hline 4 -i & $\left(d_{R} \overline{e_{L}}\right)\left(\overline{u_{L}}\right)\left(\overline{u_{L}}\right)\left(d_{R} \overline{e_{L}}\right)$ & $\# 11$ & $(\overline{\mathbf{3}}, \mathbf{2})_{-1 / 6}$ & $(\mathbf{1}, \mathbf{3})_{0}$ & $(\mathbf{3}, \mathbf{2})_{+1 / 6}$ & $\frac{1}{32}\left(\mathcal{O}_{1}\right)_{\{R R\} R}-\frac{1}{128}\left(\mathcal{O}_{2}\right)_{\{R R\} R}$ \\
& & & $(\overline{\mathbf{3}}, \mathbf{2})_{-1 / 6}$ & $(\mathbf{8}, \mathbf{3})_{0}$ & $(\mathbf{3}, \mathbf{2})_{+1 / 6}$ & $\frac{1}{24}\left(\mathcal{O}_{1}\right)_{\{R R\} R}-\frac{1}{96}\left(\mathcal{O}_{2}\right)_{\{R R\}}$ \\
\hline
\end{tabular}

Table 2. Decompositions \#4-i accidentally not listed in table 9 of the paper. 